\title{
Particularités de la prise en charge en Tunisie des séroconversions toxoplasmiques du troisième trimestre de grossesse
}

\author{
Management of Toxoplasmic Seroconversion in the Third Trimester of Pregnancy in Tunisia
}

\author{
S. Bouhlel $\cdot$ R. Ben Abdallah $\cdot$ K. Aoun $\cdot$ R. Maatoug $\cdot$ O. Souissi $\cdot$ A. Bouratbine \\ Reçu le 30 juillet 2018; accepté le 7 janvier 2019 \\ (C) Société de pathologie exotique et Lavoisier SAS 2019
}

Résumé L'objectif de notre travail était de rapporter les particularités de la prise en charge d'une série de cas de séroconversion toxoplasmique du troisième trimestre de grossesse diagnostiquée à l'institut Pasteur de Tunis. Il s'agit d'une étude rétrospective colligée au laboratoire de parasitologie-mycologie de l'institut Pasteur de Tunis entre janvier 2005 et décembre 2017, incluant 27 femmes enceintes ayant présenté une séroconversion toxoplasmique au cours du troisième trimestre de la grossesse diagnostiquée au cours de leurs suivis sérologiques. Cinq femmes ont bénéficié d'un diagnostic anténatal. La PCR (polymerase chain reaction) s'est révélée positive dans un seul cas. Deux femmes ont été mises sous pyriméthamine-sulfadoxine dont une avait une PCR positive. La spiramycine a été instituée chez 24 femmes jusqu'à l'accouchement. Une femme n'a reçu aucun traitement, la séroconversion ayant été diagnostiquée la veille de l'accouchement. Le diagnostic de la toxoplasmose congénitale a été retenu chez 20 nouveau-nés (74 \%) dont un en anténatal et 19 à la naissance. Deux étaient symptomatiques $(10 \%)$ à la naissance. Aucune manifestation clinique ou radiologique n'a été observée au cours de leurs suivis.

Mots clés Toxoplasmose · Grossesse · Séroconversion · Troisième trimestre $\cdot$ Toxoplasmose congénitale

Laboratoire $\cdot$ Tunis $\cdot$ Tunisie $\cdot$ Maghreb $\cdot$ Afrique du Nord

Summary The purpose of our study was to report the particularities of the management of toxoplasmosis seroconversion cases occurred during the third trimester of pregnancy

S. Bouhlel $(\bowtie) \cdot$ R. Ben Abdallah $\cdot$ K. Aoun $\cdot$ R. Maatoug

O. Souissi · A. Bouratbine

Laboratoire de parasitologie-mycologie,

institut Pasteur de Tunis, Tunisie

e-mail : sabrine.bouhlel89@gmail.com

S. Bouhlel $\cdot$ R. Ben Abdallah $\cdot$ K. Aoun $\cdot$ A. Bouratbine

Faculté de médecine de Tunis,

université de Tunis El-Manar, Tunisie and diagnosed in the Institute Pasteur of Tunis. The study was conducted in the Laboratory of Parasitology-Mycology of the Institute Pasteur of Tunis between January 2005 and December 2017. A total of 27 cases of toxoplasmosis seroconversion during the third trimester were included. Prenatal diagnosis was performed in five cases. PCR was positive in one case. Pyrimethamine-sulfadoxine was prescribed in one case with positive PCR and in another case as soon as maternal infection was confirmed. Spiramycine was prescribed in 24 cases for the duration of the pregnancy. One woman did not take any treatment because seroconversion was diagnosed just before delivery. Twenty newborns had congenital toxoplasmosis: 19 cases were diagnosed by serology and one case was diagnosed after amniocentesis. Two newborns $(10 \%)$ were symptomatic at birth. All the newborns had neither clinical nor radiological signs during the follow-up.

Keywords Toxoplasmosis - Pregnancy ' Seroconversion · Third trimester ' Congenital toxoplasmosis - Laboratory · Tunis · Tunisia $\cdot$ Maghreb · Northern Africa

\section{Introduction}

La survenue d'une infection toxoplasmique pendant la grossesse peut entraîner une transmission transplacentaire du parasite et exposer le fotus à des atteintes graves. Afin de réduire l'incidence de la toxoplasmose congénitale (TC), un dépistage systématique de la toxoplasmose pendant la grossesse s'impose [4]. Des stratégies de prévention variables ont été mises en place dans de très nombreux pays $[3,4]$. En Tunisie, en l'absence d'une réglementation spécifique en la matière, la sérologie de la toxoplasmose est fortement recommandée et presque systématiquement demandée lors de la première consultation prénatale. En cas de négativité de cette sérologie, les gynécologues recommandent aux femmes enceintes concernées le respect de certaines règles hygiénodiététiques et le suivi sérologique régulier et mensuel. 
Le risque de transmission toxoplasmique dépend de la date de la contamination pergravidique [17]. Avant 12 semaines d'aménorrhée (SA), le risque de passage transplacentaire ne dépasse pas les $10 \%$, mais se traduit dans la majorité des cas par une forme sévère, voire une perte fotale [16]. Inversement, après $37 \mathrm{SA}$, l'infection fœetale survient dans 66 à $76 \%$ et se traduit le plus souvent par des formes infracliniques à la naissance $[15,16]$. Une séroconversion toxoplasmique pergravidique nécessite la mise sous un traitement préventif et la recherche d'une atteinte fotale par échographie et PCR Toxoplasma sur liquide amniotique (LA) [17]. Cependant, cette démarche n'est pas systématiquement respectée lorsque l'infection survient au cours du troisième trimestre $[13,17]$.

L'objectif de notre travail était de rapporter les particularités de la prise en charge d'une série de cas de séroconversion toxoplasmique du troisième trimestre ainsi que les atteintes fœtales observées suite à ces infections.

\section{Population de l'étude et méthodes}

Il s'agit d'une étude rétrospective menée sur une période de 18 ans (janvier 2005-décembre 2017) incluant 6771 femmes enceintes, suivies ou adressées au laboratoire de parasitologie-mycologie de l'institut Pasteur de Tunis pour le dépistage systématique de la toxoplasmose au cours de la grossesse. Ces femmes ont été adressées par des médecins des secteurs privé et public. Pour les femmes séronégatives, il est recommandé une surveillance sérologique mensuelle jusqu'à un mois après l'accouchement.

La recherche des immunoglobulines IgG et des IgM a été faite par la technique Elisa (enzyme-linked immunosorbent assay) en utilisant les kits « Platelia ${ }^{\circledR}$ Toxo IgG, IgM, Biorad, France ». Devant la présence isolée d'IgM, une technique complémentaire a été pratiquée à la recherche d'IgG en utilisant le kit « Western blot Toxo II IgG LDBIO, France ».

Le diagnostic anténatal de la toxoplasmose congénitale (TC) a consisté à rechercher l'ADN parasitaire de Toxoplasma gondii au niveau du LA par la technique PCR (polymerase chain reaction) en temps réel au moyen de la technologie TaqMan. L'ADN génomique a été extrait en utilisant un kit commercial (QIAamp ${ }^{\mathbb{B}}$ DNA blood mini Kit, Qiagen, France). L'amplification a utilisé deux jeux d'amorces ciblant le gène $B 1$ et le gène cryptique «Rep $529 p b$ », et deux sondes TaqMan (TGRep, TGB1) doubles marquées FAM/TAMRA.

Le bilan sérologique à la naissance a comporté une recherche d'IgG et d'IgM par la technique Elisa, une recherche d'IgM par la technique immunosorbent agglutination assay (ISAGA) [ISAGA IgM Biomérieux, France] et l'étude des profils immunologiques comparés des IgG et des IgM par Western blot (WB) [Western blot Toxo IgG, IgM, LDBIO Diagnostics, France] entre le sérum de la mère et celui du nouveau-né $(\mathrm{NN})$ à la naissance et après en suivi entre les sérums du NN. En l'absence de critères sérologiques d'atteinte fœtale, d'autres contrôles sérologiques ont été pratiqués. Tous les NN ont bénéficié d'une échographie transfontanellaire (ETF) à la recherche d'atteinte neurologique et d'un fond d'œil (FO) à la recherche d'une choriorétinite. Une ponction lombaire a été pratiquée chez un seul NN présentant des convulsions à la naissance.

\section{Résultats}

Durant la période d'étude, 27 cas bien documentés de séroconversions toxoplasmiques survenues au cours du troisième trimestre de grossesse ont été retenus. Toutes ces parturientes ont respecté un rythme de surveillance sérologique mensuel et régulier, ce qui a permis de retenir sans équivoque la séroconversion. Vingt-quatre femmes étaient originaires du Grand-Tunis et trois provenaient de gouvernorats du Nord du pays.

Pour 25 parturientes, le diagnostic de séroconversion toxoplasmique a été retenu devant l'apparition d'IgM et d'IgG antitoxoplasmiques en Elisa lors du suivi sérologique documenté sur deux prélèvements successifs (Fig. 1). Pour les deux autres, l'apparition isolée d'IgM a justifié le recours à la technique du « WB Toxo II IgG » qui a montré trois bandes spécifiques confirmant ainsi la séroconversion.

La PCR a été pratiquée chez cinq femmes (18,5\%). La date de l'amniocentèse a été précisée pour quatre femmes (deux femmes à $30 \mathrm{SA}$ et deux à $33 \mathrm{SA}$ ). Elle s'est révélée positive dans un seul cas (20\%). L'échographie fotale était sans anomalies dans tous les cas.

Le traitement curatif à base de pyriméthaminesulfadoxine a été instauré chez une femme à la suite de la positivité de la PCR et chez une autre dès la confirmation de la séroconversion sans pratiquer une PCR. Pour 24 parturientes, la spiramycine seule a été instituée jusqu'à l'accouchement dont 20 femmes sans réaliser une amniocentèse. Une femme n'a reçu aucun traitement vu que la séroconversion a été diagnostiquée la veille de l'accouchement.

Toutes les grossesses ont été menées à terme.

Le diagnostic de TC a été retenu chez $20 \mathrm{NN}$ (74\%) ( 1 cas en anténatal et 19 à la naissance). Pour 13 NN, le diagnostic a été posé devant la présence de bandes supplémentaires en IgM et/ou en IgG au WB. La date de la positivité du WB était variable : dix cas à la naissance et trois cas à $\mathrm{j} 14$ de vie \pm 2 jours. La TC a été retenue dans cinq cas devant la présence d'IgM par la technique Elisa et/ou ISAGA au-delà de $\mathrm{j} 10$ de vie : trois $\mathrm{NN}$ à $\mathrm{j} 15$ de vie \pm 2 jours, un NN à l'âge d'un mois et demi et un autre à l'âge 


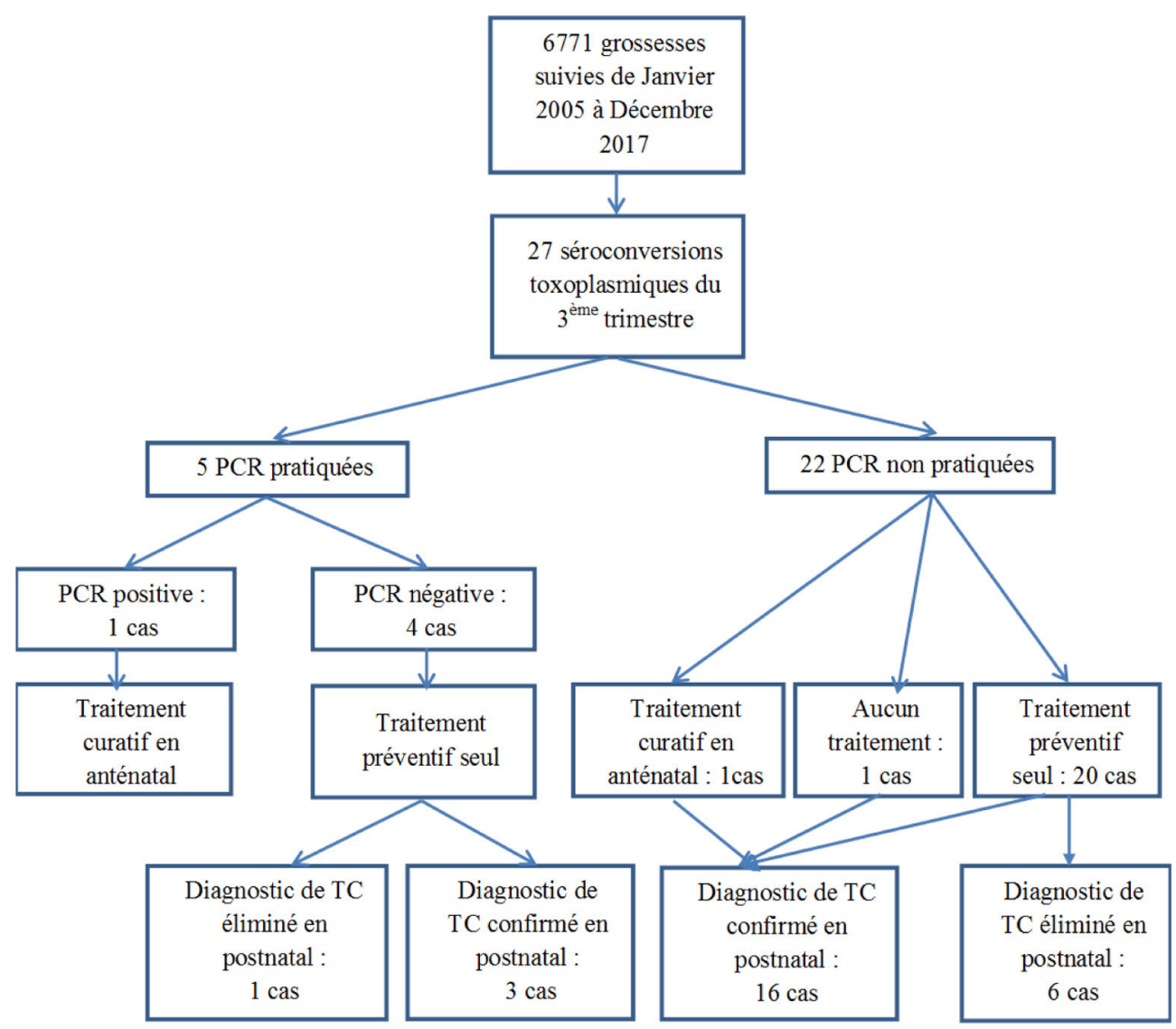

Fig. 1 Population de l'étude / Population of the study

de quatre mois et demi. Pour le dernier cas de TC, c'était la non-inflexion de la courbe des IgG qui a permis de retenir le diagnostic.

Deux NN étaient symptomatiques à la naissance (10\%). Un NN avait des calcifications intracrâniennes à l'ETF confirmées à l'IRM cérébrale. Le deuxième a présenté des convulsions et une PCR sur liquide céphalorachidien positive à $T$. gondii. Aucune manifestation clinique ou radiologique n'a été observée chez les 25 autres NN ni à la naissance, ni au cours du suivi, en particulier chez celui dont le diagnostic anténatal était positif.

À la naissance, tous les $\mathrm{NN}$ ayant une TC ont reçu un traitement curatif à base de pyriméthamine-sulfadiazine et d'acide folinique. La durée du traitement était d'un an. Le suivi thérapeutique a été assuré par la numération formule sanguine tous les mois durant la période du traitement. Un seul NN a présenté une granulopénie, ce qui a imposé une interruption transitoire du traitement. La durée du suivi s'est étalée sur une période allant de 1 an et 1 mois à 12 ans et 9 mois. Le FO a été fait tous les trois mois, les deux premières années, puis annuellement. Tous les FO étaient sans anomalies tout au long du suivi chez tous les enfants (Tableau 1).

\section{Discussion}

Le taux d'atteinte foetale était de $74 \%$ dans notre série. Ce taux confirme le risque élevé de transmission fœtale lorsque la contamination par $T$. gondii survient tardivement au cours de la grossesse $[15,16]$. Dunn et al. ont rapporté un risque de $9 \%$ de survenue d'atteinte clinique en cas de séroconversion tardive [6]. En effet, seuls $10 \%$ de nos cas étaient symptomatiques à la naissance.

En pratique courante, il n'existe pas de recommandations consensuelles quant à la prise en charge des séroconversions tardives $[13,17]$. La réalisation d'un diagnostic anténatal par amniocentèse peut être discutée ainsi que la mise sous traitement curatif d'emblée $[13,17]$. Dans tous les cas, le choix de la conduite doit être discuté entre le gynécologue, le biologiste et la patiente.

Dans notre série, l'amniocentèse a été pratiquée chez seulement cinq patientes (18,5\%). En effet, la réalisation d'une amniocentèse au cours du troisième trimestre est déconseillée par certains auteurs en raison des risques encourus [17]. Par contre, certains praticiens la réalisent jusqu'à terme [17]. Certaines études rapportent un risque de survenue de complications allant de 0,7 à $8 \%$, dont l'accouchement prématuré et la 


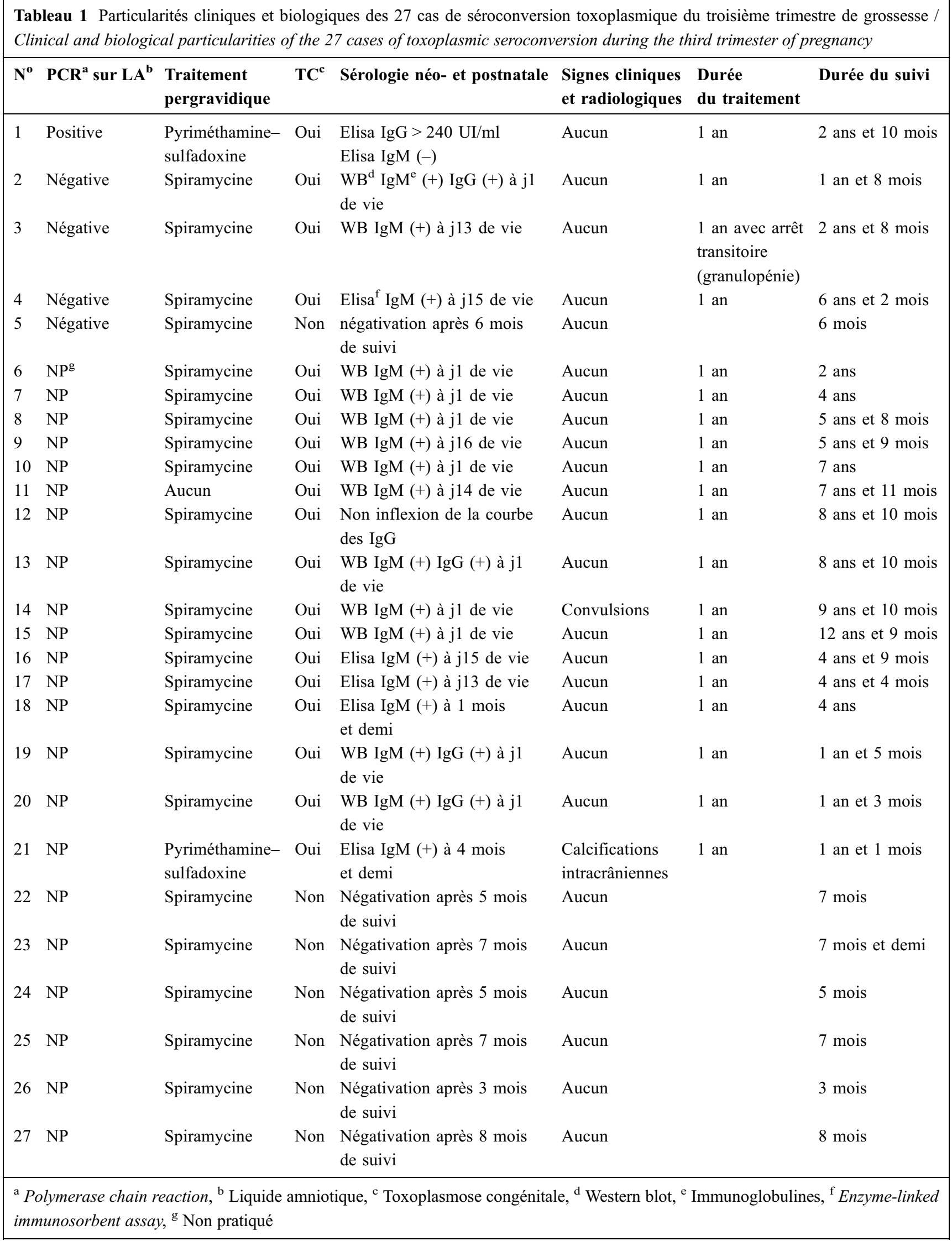


rupture prématurée des membranes [7,9,11]. Dans notre série, aucune complication n'est survenue au décours de la pratique de l'amniocentèse. Une seule PCR était positive. Le diagnostic de $\mathrm{TC}$ a été confirmé dans trois cas à la naissance parmi les quatre PCR négatives. Cela confirme qu'une PCR négative n'élimine pas le diagnostic de TC et qu'il faut impérativement contrôler les NN à la naissance [1,2]. Cette négativité pourrait être expliquée par la pratique précoce de l'amniocentèse, ne respectant pas ainsi le délai de quatre semaines après la date de l'infection maternelle. Par ailleurs, elle pourrait être expliquée par une charge parasitaire faible inférieure au seuil de détection de la méthode utilisée.

Dans notre série, une seule patiente a été mise d'emblée sous traitement curatif sans avoir recours à l'amniocentèse. Cette attitude est recommandée par certaines équipes [8,17]. En effet, elle peut être justifiée en raison du risque élevé de transmission verticale $[5,15,16]$. Dans ce cas, le diagnostic de TC a été posé en postnatal tardivement, à l'âge de quatre mois et demi. En effet, comme l'ont rapporté Bessière et al. sur une cohorte de 165 femmes ayant une infection toxoplasmique pergravidique, une diminution de la détection des anticorps antitoxoplasmiques est observée chez les NN dont les mères avaient reçu un traitement par pyriméthaminesulfadiazine, comparativement à ceux dont les mères n'ont eu que de la spiramycine [2]. Ce traitement probabiliste peut donc masquer l'apparition des anticorps chez le NN et retarder le diagnostic et donc le traitement chez ces enfants.

Pour 20 femmes de notre série, la conduite était la mise sous traitement préventif jusqu'à l'accouchement sans pratiquer une amniocentèse. Pour ces cas, la TC a été confirmée en postnatal dans $70 \%$ des cas (14 cas). En effet, l'efficacité du traitement préventif sur la transmission maternofœetale est de plus en plus discutée [16]. Selon plusieurs études, le taux de transmission n'est pas modifié par la prise d'un traitement en prénatal $[10,12]$. Cela pourrait être dû à un passage très précoce du parasite précédant le début du traitement, ce qui suggère l'intérêt d'un traitement débuté dans les deux semaines suivant l'infection maternelle [16]. Or, un dépistage même mensuel ne permet pas d'instaurer un traitement suffisamment précoce pour prévenir la transmission verticale [8].

Parmi les $26 \mathrm{NN}$ dont le diagnostic anténatal était négatif ou non pratiqué, le diagnostic de TC a été retenu sur des critères sérologiques chez $19 \mathrm{NN}$, dont $13 \mathrm{NN}$ avaient des anticorps néosynthétisés au WB. Ces résultats rejoignent les données de la littérature décrivant une meilleure sensibilité de cette technique par rapport aux autres techniques dans le diagnostic de la TC $[5,14]$.

\section{Conclusion}

Devant le taux élevé d'atteinte fotale en cas de séroconversion tardive, il est primordial de bien informer et sensibiliser les femmes séronégatives sur le risque encouru et les règles hygiénodiététiques à suivre. La prévention de la TC en Tunisie n'est pas encore réglementée malgré le risque de transmission maternofœtale surtout au cours du troisième trimestre.

Liens d'intérêts : Les auteurs déclarent ne pas avoir de liens d'intérêts.

\section{Références}

1. Bessières MH, Berrebi A, Cassaing S, et al (2009) Diagnosis of congenital toxoplasmosis: prenatal and neonatal evaluation of methods used in Toulouse University Hospital and incidence of congenital toxoplasmosis. Mem Inst Oswaldo Cruz 104:389-92

2. Bessières $\mathrm{MH}$, Berrebi $\mathrm{A}$, Rolland $\mathrm{M}$, et al (2001) Neonatal screening for congenital toxoplasmosis in a cohort of 165 women infected during pregnancy and influence of in utero treatment on the results of neonatal tests. Eur J Obstet Gynecol Reprod Biol 94:37-45

3. Blondel B, Supernant K, Du Mazaubrun C, Bréart G (2006) La santé périnatale en France métropolitaine de 1995 à 2003 : résultats des enquêtes nationales périnatales. J Gynecol Obstet Biol Reprod 35:373-87

4. Boyer KM, Holfels E, Roizen N, et al (2005) Risk factors for Toxoplasma gondii infection in mothers of infants with congenital toxoplasmosis: implications for prenatal management and screening. Am J Obstet Gynecol 192:564-71

5. Capobiango JD, Monica TC, Ferreira FP, et al (2016) Evaluation of the Western blotting method for the diagnosis of congenital toxoplasmosis. J Pediatr (Rio J) 92:616-23. doi: 10.1016/ j.jped.2016.02.014 [Epub 2016 Aug 6]

6. Dunn D, Wallon M, Peyron F, et al (1999) Mother-to-child transmission of toxoplasmosis: risk estimates for clinical counselling. Lancet 353:1829-33

7. Gabbay-Benziv R, Yogev Y, Melamed N, et al (2012) Pregnancy outcome after third trimester amniocentesis: a single center experience. J Matern Fetal Neonatal Med 25:666-8. doi: 10.3109/14767058.2011.594119 [Epub 2011 Aug 11]

8. Garcia-Meric P, Franck J, Dumon H, Piarroux R (2010) Prise en charge de la toxoplasmose congénitale en France : données actuelles. Presse Med 39:530-8

9. Geffen KT, Ben-Zvi O, Weitzner O, et al (2017) The yield and complications of amniocentesis performed after 24 weeks of gestation. Arch Gynecol Obstet 296:69-75. doi: 10.1007/ s00404-017-4408-7 [Epub 2017 May 24]

10. Gilbert R, Gras L, Wallon M, et al (2001) Effect of prenatal treatment on mother to child transmission of Toxoplasma gondii: retrospective cohort study of 554 mother-child pairs in Lyon, France. Int J Epidemiol 30:1303-8

11. Gordon MC, Narula K, O'Shaughnessy R, Barth WH (2002) Complications of third-trimester amniocentesis using continuous ultrasound guidance. Obstet Gynecol 99:255-9

12. Gras L, Wallon M, Pollak A, et al (2005) Association between prenatal treatment and clinical manifestations of congenital toxoplasmosis in infancy: a cohort study in 13 European centres. Acta Paediatr 94:1721-31

13. Haute autorité de santé (2017) Diagnostic biologique de la toxoplasmose acquise du sujet immunocompétent (dont la femme enceinte), la toxoplasmose congénitale (diagnostic pré- et postnatal) et la toxoplasmose oculaire, $79 \mathrm{p}$ 
14. Morin O (2002) Diagnostic néonatal de la toxoplasmose congénitale. Apport des nouvelles techniques de diagnostic. Immunoanalyse et biologie spécialisée 17:231-7. doi: 10.1016/ S0923-2532(02)01198-5

15. Thiébaut R, Leproust S, Chêne G, Gilbert R (2007) Effectiveness of prenatal treatment for congenital toxoplasmosis: a metaanalysis of individual patients' data. Lancet 369:115-22
16. Wallon M, Peyron F, Cornu C, et al (2013) Congenital toxoplasma infection: monthly prenatal screening decreases transmission rate and improves clinical outcome at age 3 years. Clin Infect Dis 56:1223-31

17. Yera H, Paris L, Bastien P, Candolfi E (2015) Diagnostic biologique de la toxoplasmose congénitale. Rev Franc Lab 470:65-72. doi: 10.1016/S1773-035X(15)30034-4 